Florida International University FIU Digital Commons

3-20-2012

\title{
The Interaction between Social and Physical Environments on Early Cognitive Development
}

Briana O'Dowd

Florida International University, bodo001@fiu.edu

DOI: $10.25148 /$ etd.FI12041901

Follow this and additional works at: https://digitalcommons.fiu.edu/etd

\section{Recommended Citation}

O'Dowd, Briana, "The Interaction between Social and Physical Environments on Early Cognitive Development" (2012). FIU Electronic Theses and Dissertations. 565.

https://digitalcommons.fiu.edu/etd/565 


\section{FLORIDA INTERNATIONAL UNIVERSITY \\ Miami, Florida}

THE INTERACTION BETWEEN SOCIAL AND PHYSICAL ENVIRONMENTS ON

\section{EARLY COGNITIVE DEVELOPMENT}

A thesis submitted in partial fulfillment of the

requirements for the degree of

MASTER OF SCIENCE

in

PSYCHOLOGY

by

Briana O’Dowd 
To: Dean Kenneth Furton

College of Arts and Sciences

This thesis, written by Briana O'Dowd, and entitled The Interaction between Social and Physical Environments on Early Cognitive Development, having been approved in respect to style and intellectual content, is referred to you for judgment.

We have read this thesis and recommend that it be approved.

Daniel Wright

William Kurtines

Robert Lickliter, Major Professor

Date of Defense: March 20, 2012

The thesis of Briana O'Dowd is approved.

Dean Kenneth Furton
College of Arts and Sciences

Dean Lakshmi N. Reddi University Graduate School

Florida International University, 2012 


\section{ACKNOWLEDGMENTS}

I am incredibly thankful to my supervisor and mentor, Dr. Robert Lickliter, for his outstanding guidance and encouragement throughout this project. I am also grateful for the ongoing support and contagious enthusiasm of the Developmental Psychobiology lab

team. Lastly, I thank my friends and colleagues who are a source of inspiration and healthy competition. 


\title{
ABSTRACT OF THE THESIS \\ THE INTERACTION BETWEEN SOCIAL AND PHYSICAL ENVIRONMENTS ON EARLY COGNITIVE DEVELOPMENT
}

\author{
by \\ Briana O’Dowd \\ Florida International University, 2012 \\ Professor Robert Lickliter, Major Professor
}

The impact of the relative richness or poverty of the external environment on development has been demonstrated in a variety of species and for a number of physical and cognitive processes. However, a dichotomy exists in the present literature, wherein the physical and social environments are compartmentalized. The present study investigated the potential longitudinal interaction between the social and physical environments on early cognitive development and emotional reactivity. Bobwhite quail (Colinus virginianus) were exposed to different part-time combinations of social and physical enrichment over the first 96 hours following hatch. Developmental trajectories were explored using repeated non-identical maze tasks. The experiment was inconclusive as to the effects of enrichment; however a distinct trend arose as a main effect of age. Chicks were significantly less willing to explore and solve the maze at 72 and 96 hours. Potential explanations concerning experience and physiological maturation are discussed along with sources of variability. 


\section{TABLE OF CONTENTS}

CHAPTER

PAGE

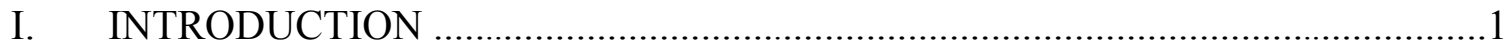

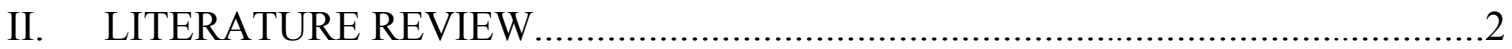

Design of the Present Study ........................................................................ 8

Research Objectives \& Hypotheses............................................................. 10

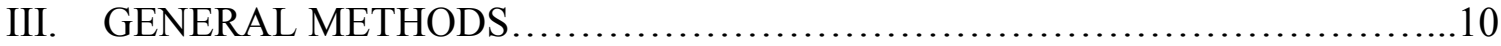

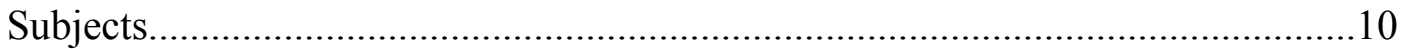

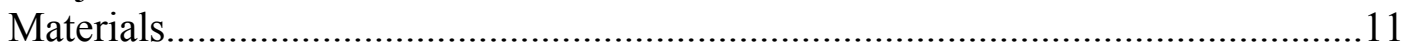

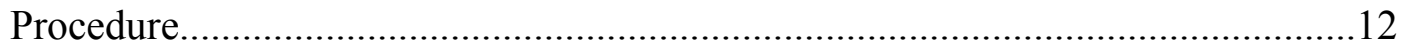

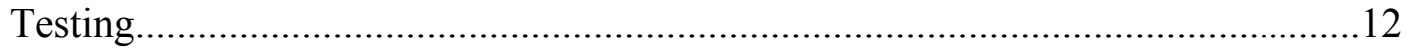

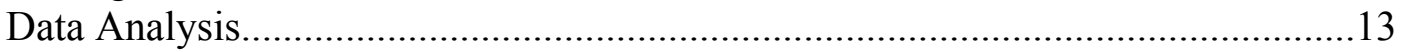

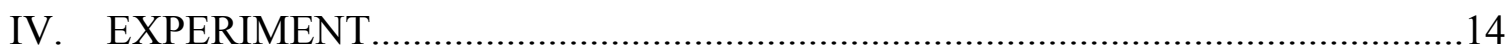

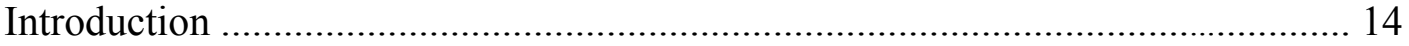

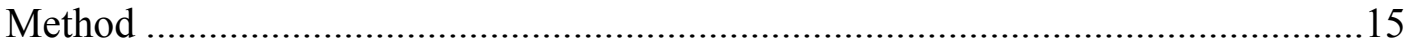

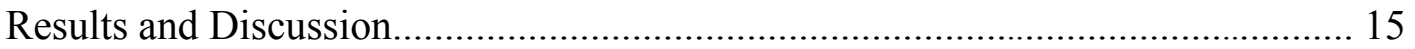

Tables and Figures.................................................... 17

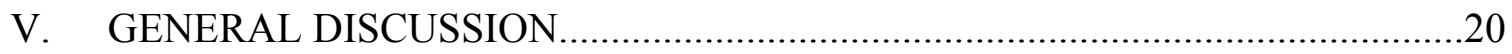

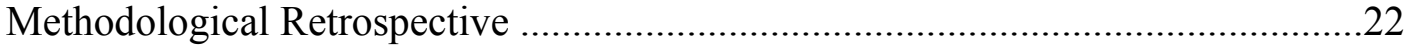

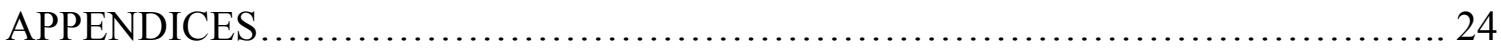

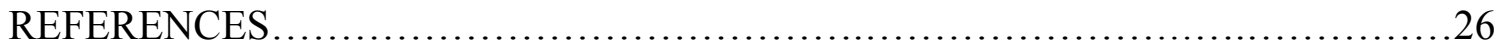




\section{LIST OF FIGURES}

FIGURE

PAGE

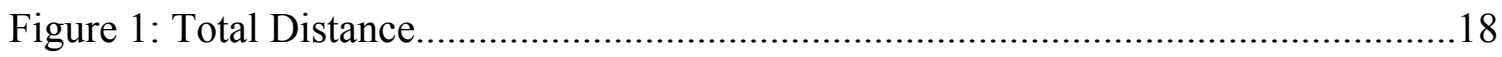

Figure 2: Time Spent Immobile........................................... 18

Figure 3: Maximum Velocity............................................ 19

Figure 4: Mean Velocity...................................................19

Figure 5: Completion Time, Unweighted...................................20 


\section{CHAPTER I.}

\section{Introduction}

Dynamic systems theories of development view development as the result of complex top-down and bottom-up interactions among levels of organization in the organism and its corresponding environment. The developmental profits from an enriched rearing environment, as well as the tragic delays resulting from an impoverished environment, have been demonstrated in a wide range of research, from neural generation in rats' brains to drastically impaired learning and maturation of human infants institutionalized in eastern European orphanages (Beckett et al., 2006). Interventions that target the external environment have the potential to set off a cascade of change, in that alterations to what is outside the organism can have effects from the behavioral to the cellular and, in turn, this influence reverberates back out systematically. Therefore, it is not surprising that studies modifying the external conditions a young organism encounters can dramatically alter their developmental trajectory. Practical implications from enrichment research include refining child-rearing practices, improving behavioral rehabilitation and recovery from neurological damage, improving animal husbandry, and ensuring appropriate behavioral and brain development in laboratory animals for a balance of control and ecological validity.

The external environment of an organism is a species specific, reciprocally determined, expanding collection, of physical, biological, and social stimuli. The external environment has been conveniently split into two domains: the physical "stuff" around the organism and the other members of the species that can interact with it. Despite the fact that most enrichment research has assumed an overarching dynamic system 
metatheory (Gottlieb, 2007), this split between physical and social environments persists in the methodology of the current, prevailing literature. A "one or the other, but not both" approach has been taken, resulting in a social versus physical perspective. However, the social and physical environments are not separate entities, but necessarily co-occur and influence each other. The following experiment explored how to best adopt an interactionist model of enrichment that recognizes a bidirectional collaboration of social and physical aspects of the environment that could impact early cognitive development.

CHAPTER II.

\section{Literature Review}

The importance of the external environment has been championed in a variety of learning and developmental theories, from Skinnerian and Piagetian, to modern dynamic systems and epigenetic perspectives. From these grand theories of development comes the idea of an active organism in an environment, its relevance determined by the species, the organism's relative maturity, and previous experience. Comparative enrichment research has demonstrated how novelty and complexity of the rearing environment can contribute to behavioral and physiological differences. A large literature focused on mammalian species, most prevalently rodents, consistently demonstrates a variety of benefits from enriched rearing. Additional evidence from historical and anecdotal human accounts indicates that the beneficial outcomes of an enriched rearing environment appear to be robust across species and developmental contexts (van Praag, Kempermann, \& Gage, 2000). 


\section{Mammals.}

The seminal work on enriched rearing stemmed from Cooper \& Zubeck's (1958) study in which providing an enriched environment to rats with poor previous maze performance enhanced future performance beyond their previously deemed "maze bright" counterparts who were housed in a standard laboratory cage. Their classic research project demonstrated that an enriched environment could not only have a compensatory effect for those subjects with a deficiency, but allowed them to thrive. A pioneering study on the neurological benefits of enriched rearing followed. Rosenzweig, Bennett, \& Diamond (1972) separated rat littler mates into either an isolated impoverished, standard laboratory, or a socially and materially enriched housing. Significant neurological gain, measured by cerebral cortex weight and neuron density, was found in rats given the enriched environment. The study served as a catalyst for a large body of developmental research on enriched rearing under the assumption that: “more experience equals a bigger brain" (Hock, 2009).

Across a variety of behavioral and maze tests as well as neurological and physiological assessments, rodents have provided a wealth of data on the benefits of enriched rearing. The introduction of a running wheel and novel objects to a standard social rearing environment resulted in mice learning a radial maze more efficiently than those without enrichment objects (Janus, Koperwas, Janus, \& Roder, 1995). Even under the stress of water deprivation, mice in the enriched condition performed better in the maze task. Using the same radial maze, Brillaud, Morillion, \& de Seze (2005), while investigating the effect of the muscarinic antagonist scopolamine hydrobromide, decided to modestly enrich the cages of their rat subjects for animal welfare purposes. The rats 
were socially isolated, as required by their methodology to study the drug, but now received corn chips and a plastic tube. These simple materials serendipitously led to an increase in exploratory behavior, and Brillaud et al., (2005) decided to adopt this housing strategy as standard care. On the neurophysiological front, material environmental enrichment has been shown to contribute to a differential distribution of normal levels of a neurotrophin protein, which is associated with emotional behaviors (Zhu et al., 2006).

Social contact has been shown to be a complimentary enrichment procedure to material enrichment in social rodents. The social isolation of rats accompanied with a barren cage for the post-weaning rearing environment has been shown to enhance activity in the subsequent introduction of a novel stimulus, whereas an enriched social environment made habituation to novelty quicker (Schrijver et al., 2002). Additionally, social isolation has been shown to increase novel object and food preference (Hall et al., 1997a; Hall et al., 1997b). The absence of conspecifics has also been shown to impair performance on spatial learning in the Morris water maze (Wongwitdecha \& Marsden, 1996). Prolonged isolated rearing conditions have been shown to contribute to the display of fear and depressive-like behaviors in rats, such as hyperactivity during an open field task (Molina-Hernandez, Tellez-Canatara, \& Perez-Garcia, 2001).

Additionally, enriched rearing research in non-human primates has provided potential insight into how humans may react to early enrichment. The rhesus monkey (Macaca mulatta) has been widely used as an analogue to the human brain in neurocognitive research. For example, Sanchez et al., (1998) found that rhesus monkeys reared in an enriched, semi-naturalistic social environment versus a laboratory nursery had significantly thicker corpus callosum size and performed better on a variety of 
cognitive tasks. Research conducted at the Wisconsin Primate Laboratories has explored the enriched rearing paradigm in the rhesus monkey. Massed together, these studies suggest that stimulus deprivation during development can produce monkeys that are inactive, lack spatial exploration behavior, and are socially withdrawn (Sackett, 1965).

Other work with mammals has gained attention in both academia and in the media, as the impact of enrichment of the rearing and housing conditions of livestock has implications for USDA animal welfare regulations. For example, piglets were given a tonic immobility measure of emotional reactivity and tested in a T-maze. After being categorized into two groups based on reactivity, piglets received either a barren rearing environment or one enriched with a straw substrate. Piglets who were originally classified as highly emotionally reactive and placed in the enriched rearing condition demonstrated improvement over their counterparts (Bolhuis et al., 2004). In a separate agricultural enrichment investigation, ewes were exposed to human interaction as well as colorful moving objects during a sensitive period between giving birth and weaning of their lamb (Vandenheede \& Bouissou, 1997). Ewes in the enriched environment subsequently displayed less fearful behaviors during isolation and surprise tests when compared to controls in standard housing.

Avian species.

The convenient prenatal packaging and precocial nature of many different avian species allows for earlier and more efficient manipulation of the external environment during early development. Enrichment research in avian species has mostly concentrated on addressing commercial and agricultural concerns, such as enhancing tameness and productivity. For example, presenting novel, colorful, complex, movement-laden stimuli 
on a television screen has been shown to reduce fearfulness and increase egg-laying in domestic chickens (Gallus gallus) (Jones, 2002). Additionally, zoological and laboratory concerns of avian husbandry and welfare have concentrated on achieving ecological validity through appropriate environmental enrichment (Hawkins et al., 2001). Precocial avian species offer a unique experimental opportunity in that immediately after hatching they are able to be an active agent within a broad external environment, unlike rodents or other altricial mammals that are born without fully developed sensory systems. Therefore the external environment can be manipulated at hatch before other early postnatal history is constructed.

The importance of social stimulation for the perceptual and cognitive development of precocial birds, such as ducklings, domestic chicks, and quail chicks, has been firmly established in the literature (Lickliter, Dyer, \& McBride, 1993). In social or restricted physical isolation, developmental delays have consistently been demonstrated. Likewise, social enrichment (enrichment versus crowding) has been shown to facilitate learning. Socialization of precocial avian species is essential for the birds to learn species-typical behaviors and perceptual cues, such as maternal calls (Lickliter, Dyer \& McBride, 1993). Several studies indicate that both social and physical enrichment are key to emotional regulation and optimal development in social avian species. For example, Francois, Mills, \& Faure (1998) found that young Japanese quail (Coturnix japonica), from 2 days to 6 weeks old, prefer social enrichment over physical enrichment and access to food and water. Given a simultaneous choice, Japanese quail spent the majority of their time (over $80 \%$ ) with the social group, $10 \%$ of the time to access nourishment, and the remaining $10 \%$ investigating the enrichment material, a simple 
substrate. In a study by Valance et al., (2007), quail were raised with a cage-mate and a familiar inanimate object, a ball. Results indicated that removal of the cage-mate or the ball and subsequent introduction of a novel social or material stimulus resulted in behaviors typical of negative affect and an increased heart rate, indicating that changes in either the social and physical environment can produce a similar arousal response.

A series of environmental enrichment experiments by Miller \& Mench (2005) explored how four different forms of environmental enrichment (foraging opportunities, structural complexity, sensory stimulation/novelty, and social companionship) contribute to different behavioral outcomes in adult Japanese quail. The different forms of environmental enrichment seemed to interact to contribute to differential behavioral budgets. Physical enrichment items increased overall activity and foraging, and structural complexity together reduced anxious pacing behavior and fear responses. On the other hand, increased social contact reduced the amount of time subjects spent with the physical enrichment items.

In a number of different bird species, the introduction of environmental complexity and novelty, when controlling for social contact, has resulted in a decrease in negative affective behaviors and increases in spatial exploration (Francois, Mills, \& Faure, 1998; Freire, Cheng, \& Nicol, 2004; Lazic, Schneider, \& Lickliter, 2007; Miller \& Mench, 2005). For example, providing bunches of string encouraged foraging and reduced self-injurious behavior and aggression in adult domestic chickens, both in a laboratory and commercial farm setting (Jones, 2002). A study of crimson-bellied conures (Pyrrhura perlata perlata) who had deleterious feather-plucking behavior prior to intervention, showed a decrease in plucking and other anxious behaviors after the 
administration of natural enrichment items and food options (van Hoek \& King, 1997). Providing objects that allow the opportunity to hide in the rearing environment of the young domestic chick has been shown to increase spatial navigation skills (Freire, Cheng, \& Nicol, 2004). The introduction of burrowing tubes to a standard laboratory rearing tub resulted in an increase in spatial exploration in a subsequent maze task in bobwhite quail chicks (Lazic, Schneider, \& Lickliter, 2007).

\section{Design of the Present Study}

The present study was designed to address several gaps in the current enrichment literature by using a part-time enrichment, micro-longitudinal approach. As mentioned previously, prolonged social isolation will typically result in developmental delays and increased emotional reactivity in precocial birds. However, in order to study the interaction between the social and physical aspects of the environment, the social context in which presentation of novel objects occurs must be manipulated. Therefore the present study adopted a "part-time" alternate environment methodology in which quail chicks were housed in a standard social laboratory tub, except for a two-hour condition session each day for the first three days after hatching. Previous research using a part-time enrichment procedure has been shown to have a significant effect on quail chicks' emotional reactivity and cognitive development, although gains are considered milder with those given full-time enrichment exposure (Bechor, Herrington, \& Lickliter, 2010).

Current enrichment research typically focuses on the outcome of prolonged exposure to alternative levels of social or physical enrichment (Hock, 2009). There are two major deficiencies in the current study of enriched rearing. Firstly, current methodology has separated enrichment as either social or physical, ignoring the obvious 
possibility of an interaction between the two aspects of the environment that necessarily co-occur. While subjects in previous research have been socially isolated, socially enriched, or simultaneously socially and physically enriched throughout development, no study has provided enrichment to the isolated individual or has sufficiently controlled for the known detrimental effects of prolonged isolation. Secondly, to date, no enrichment study has been conducted that tracks the differential developmental trajectories that arise as experience with the external environment is compounded over time. Simple pre- and post-enrichment paradigms have overpopulated the literature, but none have looked into the trajectory, the process by which experience impacts emotional reactivity and cognitive ability over early development. The past methodology has perpetuated the notion of an outcome variable versus an interactive developmental process. Given the suggested neurobiological underpinnings that result from enrichment and give rise to behavioral differences, gradual change over time is required. Therefore, development, as change over time, should be studied longitudinally. The current pre- and post-test methodology is inadequate to describe how this process may occur.

The present experiment was designed to begin to address these deficiencies by: (a) investigating the interaction between the social environment and the physical environment on cognitive ability and emotional reactivity, while controlling for the known adverse effects of complete social isolation and (b) assessing how any observed effect progresses longitudinally over the first 96 hours of life in the bobwhite quail chick. 


\section{Research Objectives and Hypotheses}

Objective \#1: Determine if there is a moderating [interaction] effect between the early postnatal social and physical environments on quail chicks' emotional reactivity and cognitive ability.

Objective \#2: Track individual developmental trajectories that result from increasing exposure to alternative environments early in life.

Hypothesis \#1: Bobwhite quail neonates exposed to an enriched environment in a group setting will display less emotional reactivity in subsequent novel maze tests versus their counterparts who receive physical enrichment while socially isolated.

Hypothesis \#2: Bobwhite quail neonates exposed to an enriched environment in a group setting will perform more efficiently in novel maze tests versus their counterparts who receive enrichment while socially isolated.

Hypothesis \#3: As development and exposure to alternative environments increases and compounds over time, the differences between group enriched chicks, isolated enriched chicks, and control chicks will become more evident.

\section{CHAPTER III.}

\section{General Methods}

\section{Subjects}

Subjects were 180 incubator reared bobwhite quail chicks (Colinus virginianus). Fertilized, unincubated eggs were received weekly from a commercial game bird supplier and set in a custom-built incubator maintained at $37.5^{\circ} \mathrm{C}$, and a relative humidity of 75 $80 \%$. Following hatching, chicks were transferred in same age groups of 13 chicks into standard plastic rearing tubs in a general rearing room maintained at approximately $30^{\circ}$ 
C. Chicks had constant access to water and commercial feed in the standard rearing tub and in the alternative environment tubs, but not during the maze trials. Approximately twelve hours after hatching, chicks were leg banded with a uniquely colored paper and wire twist-ties.

Materials

Apparatus

Behavioral tests were conducted using four different $60 \mathrm{~cm}$ by $60 \mathrm{~cm}$ MDF square mazes set inside a sound attenuated room (see Appendix A). A video camera was mounted directly above the maze for remote observation and data collection in an adjoining room. A small speaker concealed outside of the maze was used to present auditory stimuli during the trials. Additionally, a $12 \mathrm{~cm}$ by $12 \mathrm{~cm}$ wire mesh box housed six same-age chicks at the end of the maze. Portable space heaters were also be arranged to encourage movement toward the maze end. Maze difficulty was controlled by using the same size and number of walls in each.

\section{Enrichment Items}

A variety of physical enrichment items were presented to chicks in two of the four experimental conditions. Seven new items were used during each of three enrichment sessions. Enrichment items were chosen to include a range of exploration opportunities, including objects to hide in or under, objects that can be moved by the chicks, reflective objects, objects with flavors, and objects that produce sound. A detailed list of enrichment items and their presentation schedule is included in Appendix B. 


\section{Procedure}

Bobwhite quail chicks were divided into four experimental conditions following hatching: (1) a group enriched condition, wherein chicks were placed in the physically enriched environment in groups of nine (2) an isolated enriched condition, wherein chicks were placed into identical physically enriched environments in social isolation (3)

a group control condition, in which chicks were transferred into another standard rearing bin to account for handling, and (4) an isolated control condition, in which chicks were separated into standard rearing bins in social isolation. All placements into the alternate environment (either enriched or standard control) lasted for 2 hours, with the first session occurring approximately 24 hours after hatching and baseline testing. After the two hour session finished, chicks were returned to a mixed-condition laboratory standard rearing bin. Therefore, the chicks in the isolated conditions were only alone for the 2 hour session each day, and were then reunited with their brood mates.

After $1300 \mathrm{~h}$ subjects were transferred into their alternate environment conditions for two hours. A cool-down period of at least 3 hours separated the maze trials and the alternative environment sessions in order to account for any effects that resulted from post-test arousal. Each chick will receive the same assigned condition at 24, 48, and 72 hours of age. Thus, by the $96 \mathrm{hr}$ maze test, subjects will have received three 2-hour altered environment sessions.

\section{Testing}

All chicks were tested individually at 24, 48, 72, and 96 hours of age. The $24 \mathrm{hr}$ maze served as a baseline test before any separation and/or enrichment occurred. One of the 4 different mazes was arbitrarily assigned to each developmental point. Therefore, 
each chick went through the testing procedure 4 times, each with its own unique maze configuration. All maze testing occurred before 10am each day. A subject was placed in the maze in a small opaque plastic start cup. At the end of the maze a small wire mesh box contained 6 same-aged chicks. Additionally, the corresponding quadrant of the end zone presented a bobwhite maternal assembly call, played from a concealed speaker, and two space-heaters that provided an attractively warm temperature gradient, as precocial chicks are unable to independently thermoregulate at the ages studied in the current experiment (Whittow \& Tazawa, 1991). After a brief acclamation period, the chick was released by lifting the start cup and all subsequent movements were recorded for 300 seconds. Measurement was conducted using a computer animal tracking software, EthoVision XT by Noldus.

Data Analysis

The dependent variables of emotional reactivity and cognitive ability were operationally defined and measured as maze movement and maze completion, respectfully. Measures of emotional reactivity were: 1) total distance moved, 2) time spent immobile, 3) mean velocity and 4) maximum velocity. Measures of cognitive ability were: 1) maze completion (dichotomous) and 2) time to maze completion. All test trials ended when 300s had elapsed, giving chicks that finished the maze early equal opportunity to explore the maze. Each subject was tested at $24 \mathrm{hr}$ (baseline), $48 \mathrm{hr}, 72 \mathrm{hr}$, and $96 \mathrm{hr}$ - creating a sufficient number of time points to describe the developmental trajectories of each measure. Chicks that spent more than 290s immobile during each of the four maze trials were considered habitual non-responders and were excluded from the 
final data analysis. The proportion of non-responders between the four conditions was evaluated using a chi-square test.

Scores of total distance, time spent immobile, mean velocity, and maximum velocity were evaluated using individual repeated-measures Analysis of Variance. Responses were compared for their overall trajectory (age*condition). At each individual time point, time until maze completion was evaluated using one-way ANOVAs and differences proportion of chicks that finished the maze between the four conditions was evaluated using Chi-square tests. Main effects for age and condition were evaluated using a one-way ANOVAs and post-hoc Bonferroni comparisons. Significance levels of $p<.05$ were used to judge all results.

\section{CHAPTER IV.}

\section{Experiment}

\section{Introduction}

To investigate the longitudinal effects of compounded exposure to socially and physically enriched environments on emotional reactivity and cognitive development, newly hatched quail chicks were repeatedly exposed to one of four part-time alternative rearing conditions: group enriched, isolated enriched, group control, or isolated control. In line with previous enrichment research, I hypothesized that the chicks that were given physical enrichment within a social context (group enriched) would show less emotional reactivity and have greater cognitive gains than their isolated enriched counterparts.

Likewise, chicks that were socially isolated without physical enrichment would perform the poorest, in keeping with the known detrimental effects of social isolation in early development. Furthermore, I hypothesized that over time the increasing exposure to 
different part-time rearing conditions would result in increasingly distant developmental trajectories across the four groups as they aged.

Method

One-hundred and eighty bobwhite quail chicks, divided into four conditions (group enriched $n=60$, isolated enriched $n=30$, group control $n=60$, isolated control $n$ $=30)$ served as subjects. All subjects were leg-banded with colored twist-ties for individual identification and were housed in a laboratory standard "home" bin in a 13 chick cohort. Out of each cohort nine chicks were assigned to a group condition together and four were isolated. For two hours each day at approximately 24, 48, and 72 hours post-hatch, chicks were transferred from the home bin into their assigned condition. The group enriched condition received novel objects with their broodmates, the isolated enriched condition received identical novel objects while socially isolated, the group control condition was transferred into a laboratory standard bin in order to account for any effect of human handling, and the isolated control condition was separated into socially isolated standard bins. All chicks were tested using maze tasks at 24 hours before any exposure to their assigned condition (baseline) and then at 48, 72, and 96 hours (see General Methods for details).

\section{Results and Discussion}

Results are summarized in Tables 1-3. There was no significant difference between the proportion of non-responders between the four groups $\left(\chi^{2}(3)=2.014, p=\right.$ .569). After removing the non-responders, which represented $18 \%$ of the overall sample, the sample size for the subsequent analysis was as follows: group enriched $n=47$, isolated enriched $\mathrm{n}=24$, group control $\mathrm{n}=50$, isolated control $\mathrm{n}=27$. Mauchly's test 
indicated that the assumption of sphericity had been violated across all measures included; therefore degrees of freedom were corrected using the Greenhouse-Geisser estimates of sphericity for the repeated-measures ANOVAs.

Across all measures of emotional reactivity there was no significant difference found between the trajectories (age* condition) of the four conditions: total distance $(\mathrm{F}(7.9,379.6)=.430, \mathrm{p}=.901)$, time spent immobile $(\mathrm{F}(7.6,429)=.407, \mathrm{p}=.910)$, mean velocity $(\mathrm{F}(7.9,379.5)=.434, \mathrm{p}=.899)$, and maximum velocity $(\mathrm{F}(7.2,347.8)=.403, \mathrm{p}$ $=.906)$. There were no significant differences in the proportion of chicks that completed the maze between groups at any time point: $24 \mathrm{hr}\left(\chi^{2}(3)=1.185, p=.756\right), 48 \mathrm{hr}\left(\chi^{2}(3)=\right.$ $.645, p=.886), 72 \mathrm{hr}\left(\chi^{2}(3)=3.754, p=.289\right)$, or $96 \mathrm{hr}\left(\chi^{2}(3)=2.845, p=.416\right)$. There was an observed main effect for age for maze completion $(F(3,592)=8.587, p<.001)$ and maze completion time $(F(3,592)=2.937, p=.032)$. Bonferroni tests found that chicks at 48 hours were significantly more likely to finish the maze and finished the maze faster than at any other time point. However, at 72 and 96 hours, chicks' maze performance was not significantly different than baseline. Overall there was a high amount within-group variability - variability which prevented a seemingly obvious graphical trend from reaching significance. The potential sources of this variability are discussed below. 
Table 1: Non-Responders

\begin{tabular}{cccc} 
Condition & Total n & Non-Responders & Percent \\
\hline Group Enriched & 60 & 13 & $21.6 \%$ \\
Isolated Enriched & 30 & 6 & $20.0 \%$ \\
Group Control & 60 & 10 & $16.6 \%$ \\
Isolated Control & 30 & 3 & $10.0 \%$ \\
\hline
\end{tabular}

Table 2: Maze Completion by Age

\begin{tabular}{ccccc} 
Age & N & Finishers & Percent & $\begin{array}{c}\text { Finish Time } \\
(\mathbf{s})\end{array}$ \\
\hline 24 & 147 & $66^{\mathrm{a}}$ & $44.9 \%$ & $151.9^{\mathrm{a}}(80.8)$ \\
48 & 147 & $85^{\mathrm{b}}$ & $57.8 \%$ & $112.6^{\mathrm{b}}(81.7)$ \\
72 & 147 & $46^{\mathrm{a}}$ & $31.3 \%$ & $118.7^{\mathrm{a}}(80.1)$ \\
96 & 147 & $52^{\mathrm{a}}$ & $35.4 \%$ & $126.8^{\mathrm{a}}(91.5)$ \\
& & & \\
\hline
\end{tabular}


Figure 1: Total Distance

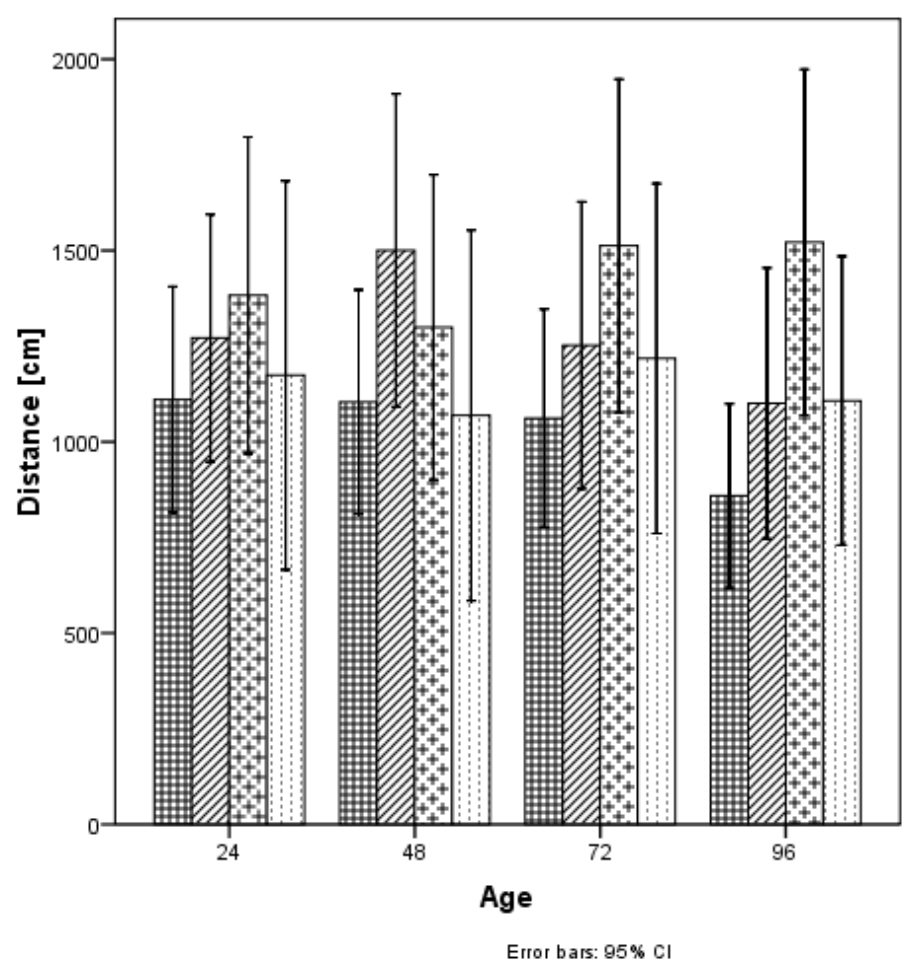

Condition

围Group Control

Q Group Enriched

TIsolated Control

Isolated Enriched

Figure 2: Time Spent Immobile

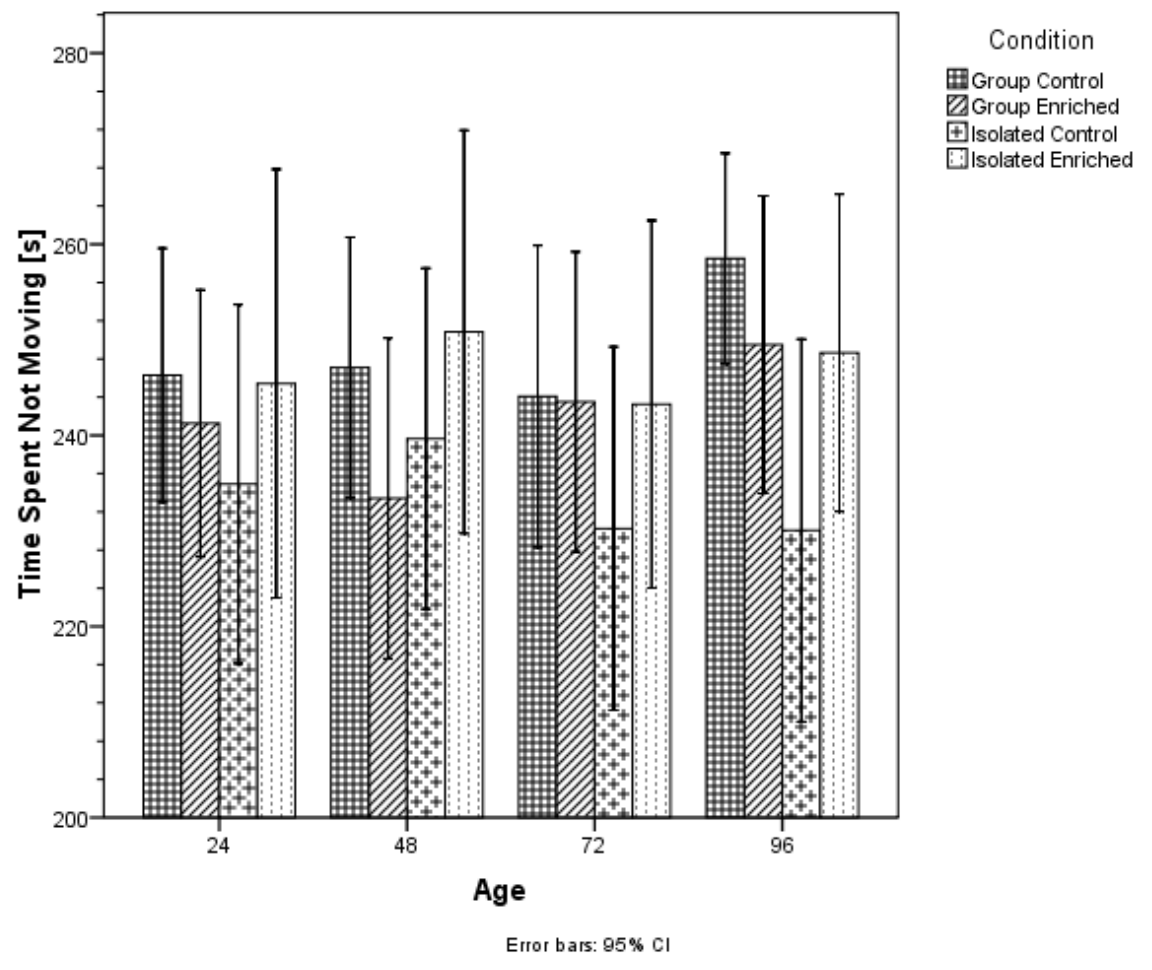


Figure 3: Maximum Velocity

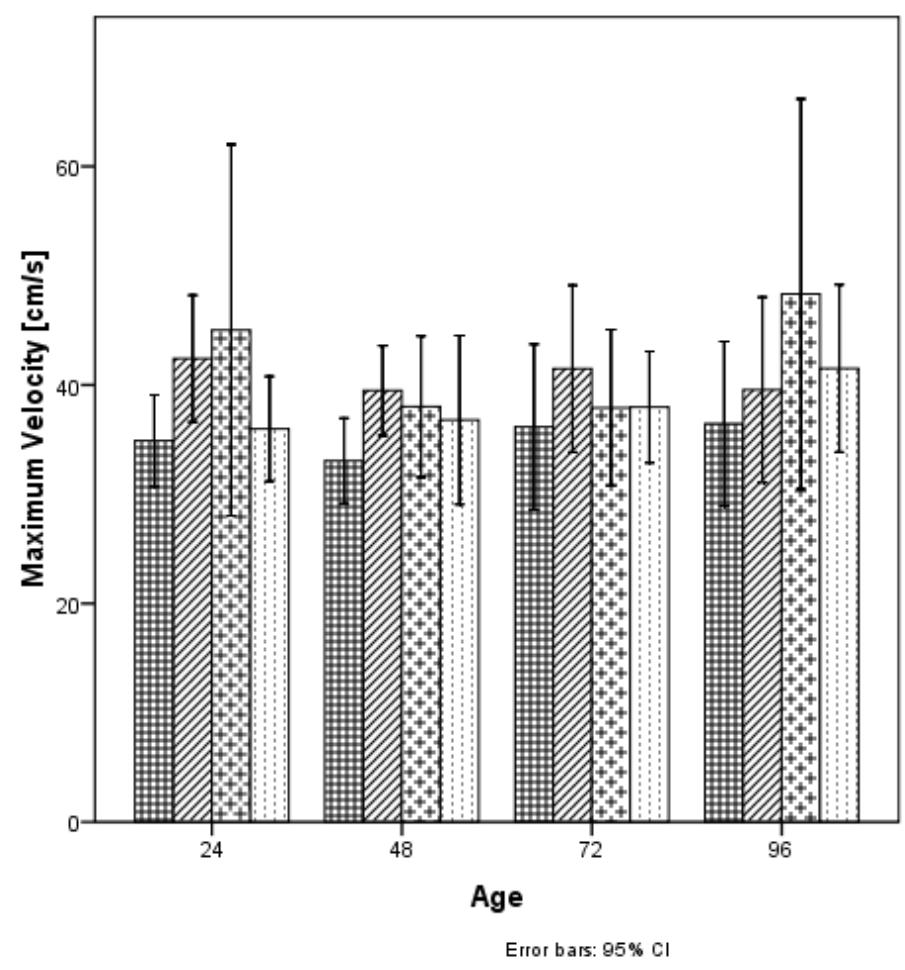

Group

围Group Control

WGroup Enriched

田Isolated Control

Bisolated Enriched

Figure 4: Mean Velocity

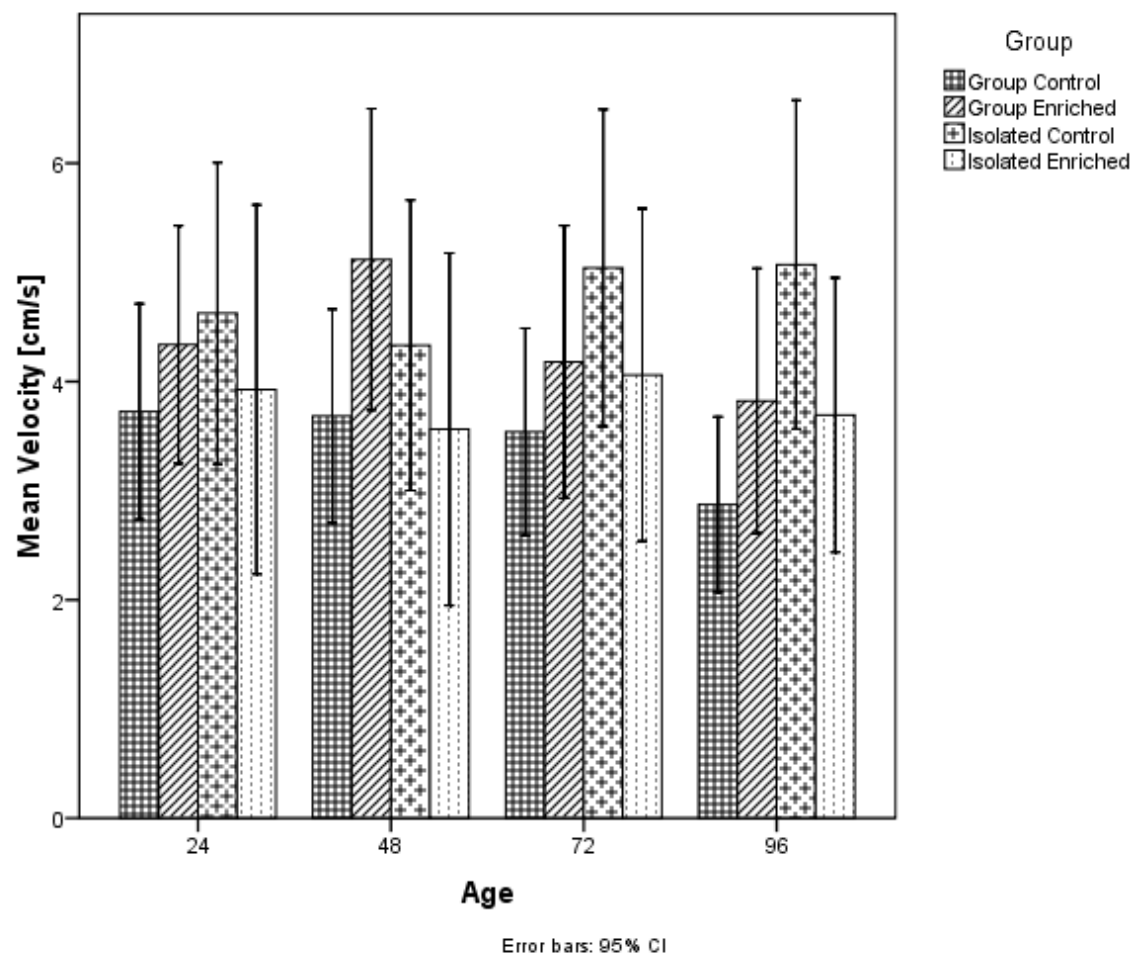


Figure 5: Completion Time, Unweighted

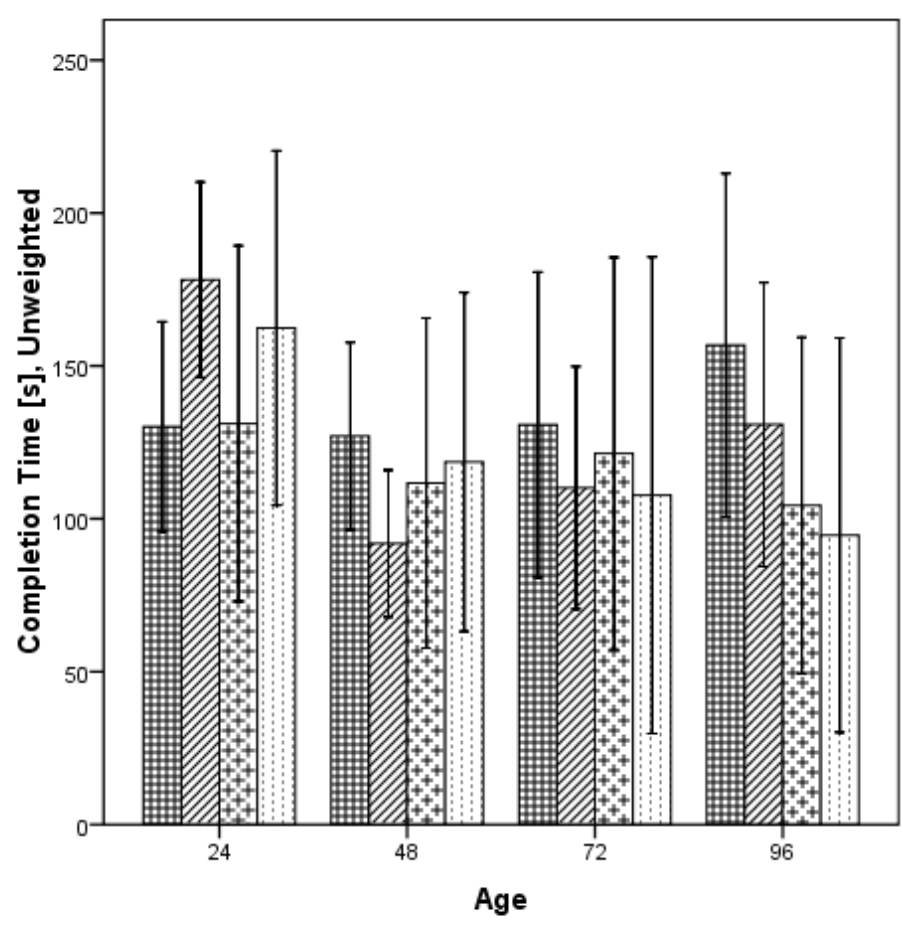

Condition

田Group Control

Group Enriched

†Isolated Control

Disolated Enriched

Error bars: $95 \% \mathrm{Cl}$

CHAPTER $V$.

\section{General Discussion}

The main goal of the present study was to explore the developmental trajectories of emotional reactivity and cognitive ability in chicks exposed to different combinations of social and physical enrichment following hatching. While there were no significant differences between the experimental conditions, there were significant differences in chicks' performance as a result of age. Perhaps accounting and controlling for the large amount of within-group variability can help disentangle any potential effect of the enrichment protocol from other factors. Some of the potential sources of variability include hormone regulation and arousal due to the time of sunrise in the rearing room (Hazard, Couty, Faure, \& Guemene, 2005), as subjects were tested in the morning and the experiment included both summer and winter months. Therefore some cohorts of 
subjects had several hours of sunlight prior to testing at $0800 \mathrm{~h}$ and others had to be artificially woken up with a florescent light only about 30 minutes before testing. Unfortunately, cohort or batch was not able to be controlled for in the final analysis, since not all experimental conditions were present in each batch. Additionally, the amount of exposure to the alternative environments might not have been sufficient to warrant a significant difference between groups. It was the intention of the present study to minimize the amount of time the isolated enriched/isolated control chicks were away from their broodmates, because to the well-documented detrimental effects of isolation, and only manipulate the context in which the novel objects were presented. However, this resulted in a very limited 2-hour window that chicks were exposed to the physical enrichment. The choice of objects may have had an impact on spatial ability and emotional reactivity. Although the objects were items that served many different functions such as the opportunity to hide or engage reflective surfaces, the objects were not things the chicks would not normally encounter in a more ecologically valid setting. Additionally, given the arousal created by handling and transferring them, the chicks may have not been given enough time to calm down and benefit from the novel objects. Along with determining the sources of variability, investigating the threshold and effect size of different durations spent in alternative environments is an obvious next step in determining if and how this interaction plays out over development.

The longitudinal design was able to capture an interesting segment of the developmental process of the willingness to explore, which may have practical implications for future testing. At 48 hours chicks were more likely to finish the maze and finished the maze more efficiently than at baseline. The observed trend neatly 
corresponds with several developmental events. Around 48 hours, the quail chick's reserve of yolk is diminishing and the chick must start finding food and water. Therefore, the chick's need to find resources may encourage it to investigate its environment. However, the subsequent decline of exploration at 72 and 96 hours is counterintuitive to this explanation; at 72 and 96 hours, the completion rates are not significantly different from baseline. The loss of maze efficiency at 72 and 96 hours may partially be the result of a hormone regulating system, the hypothalamic-pituitary-adrenal (HPA) axis, becoming functional. The HPA axis, found in mammals and avian species, regulates levels of corticosteroids and influences fear responses and may inhibit exploratory behavior and efficient spatial navigation (Debonne et al., 2008).

On the behavioral level, lab-reared chicks at 72 and 96 may have learned that food and water are constantly provided in the rearing bin and, in the present study, the chicks may have become accustomed to being returned to the bin after their short maze trial. In the same vein, the repeated maze trails may have resulted in testing habituation or fatigue. This second explanation is somewhat less convincing, as other post-test only studies in the lab have a similar lack of responding at 96 hours. The implications for the results are limited but nevertheless practical for future research, particularly with precocial avian species.

\section{Methodological Retrospective}

The design of the present study aimed to operationally separate the social and physical environments in order to manipulate both simultaneously to investigate a possible interaction. The manipulation of the current experiment was further complicated by attempting to minimize the known damaging effects of complete social isolation by 
using an extremely low threshold of exposure to alternative environments, compared to previous research. In retrospect, the short duration subjects spent in the conditions may have been too brief to make a significant difference, with a mere total of 6 hours of exposure amassed by 96 hour testing. Increasing the duration of enrichment to one forth to one half of total rearing time may have an impact on emotional reactivity and cognitive development. Alternatively, a different full-time housing apparatus could be devised to provide social and physical enrichment in separate containers that the subject can freely move between, therefore manipulating the context in which novel objects are presented, but still providing much-needed social interaction. However, as demonstrated in Francois, Mills, \& Faure (1998), birds are likely to spend the majority of their time with the social group, defeating much of the intended purpose of the manipulation.

In retrospect, measurement could have been conducted differently as to include several more testing paradigms of emotional reactivity and cognitive ability, both active and passive on the part of the chick. While traditional maze testing is able to concurrently evaluate several aspects of emotional reactivity and cognitive development, it is dependent on the chick's willingness to explore. Another test that does not depend entirely on this willingness, such as tonic immobility, could be included in addition to the traditional maze task. In order to account for some of the within-group variability, additional measures such as body weight and fecal cortisol levels could also be considered. To control differences between individual hatches, each condition could be represented in each cohort. Lastly, to minimize potential variability due to egg size/quality and arousal due to sunrise, all subjects should be hatched and tested within a single season. 


\section{APPENDICES}

Appendix A: Mazes

$24 \mathrm{hr}$ [baseline] maze:

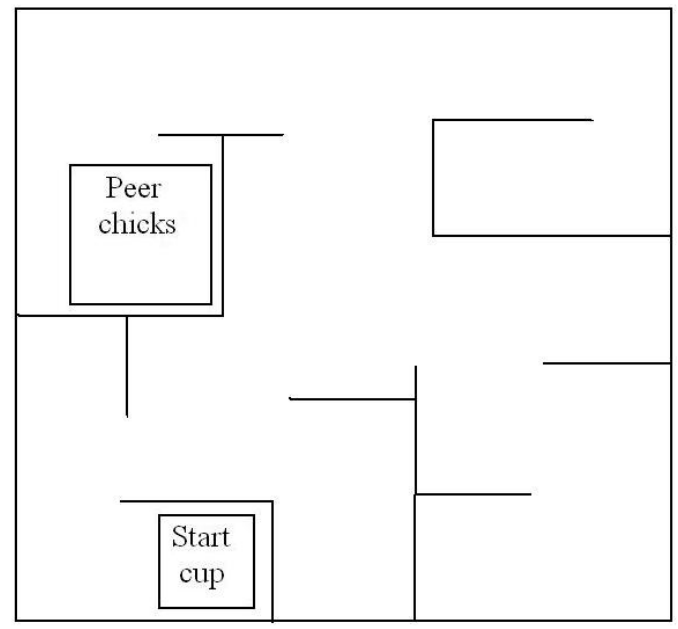

$72 \mathrm{hr}$ maze:

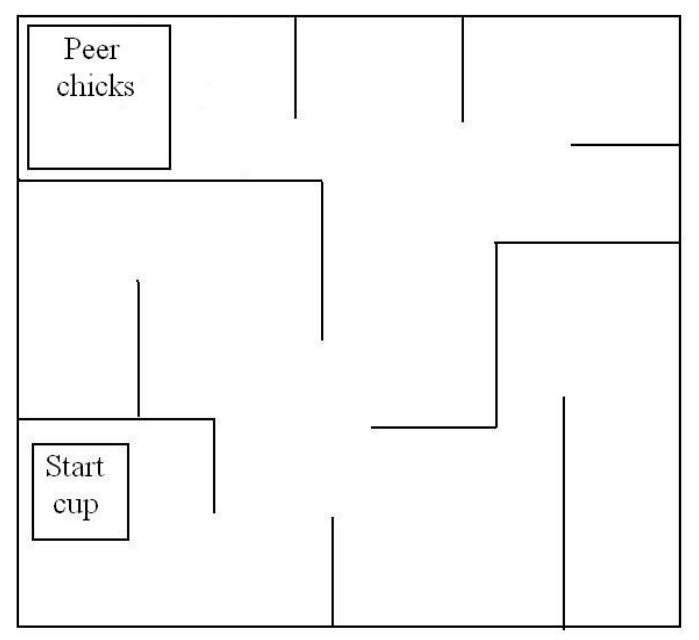

48 hr maze:

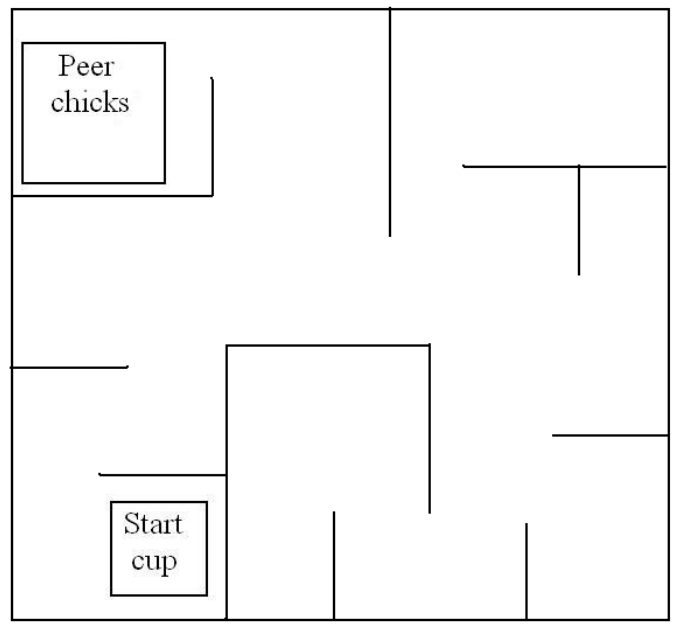

$96 \mathrm{hr}$ maze:

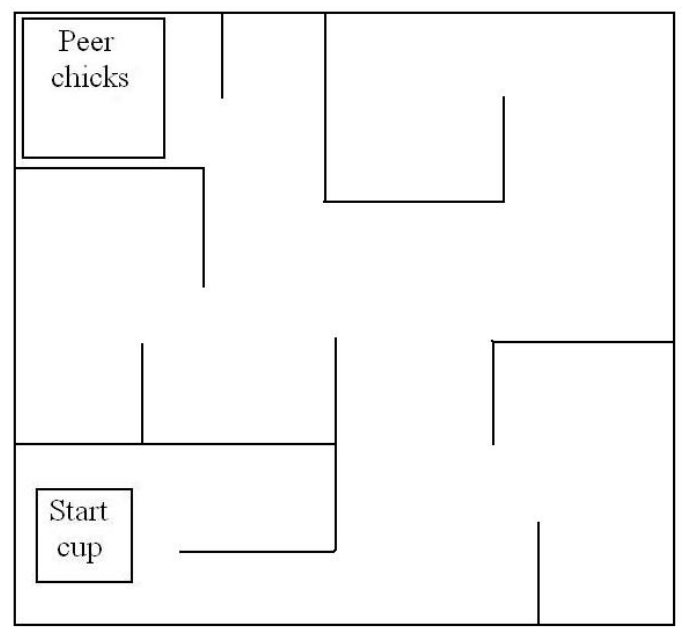




\section{Appendix B: Physical Enrichment Items}

Items are placed inside a standard rearing bin along with feed and water. Items are placed before chicks are transferred into the alternate environment. All chicks in the physically enriched environment conditions receive the same items on the same day in approximately the same configuration. Items are all disposable to control for wear and sanitation. All items are non-toxic and commercially available.

$24 \mathrm{hr}$ items:

-half tennis ball

-color pipe cleaner

-4 " felt square

-parrot toy with bell

-wooden dowel

-cotton ball

-iridescent marble

$48 \mathrm{hr}$ items:

-cardboard tube

-1 " round mirror

-twine

-wooden alphabet block

-large color turkey feather

-stainless steel spoon

-fruit-flavored wooden parrot toy

$72 \mathrm{hr}$ items:

-plastic Solo cup

-1 " reflective glass bead

-sock

-jingle bell

-ribbon

-color plastic ball

-tissue paper 


\section{REFERENCES}

Bechor, M., Herrington, J., \& Lickliter, R. (2010). Amount of enriched rearing affects bobwhite quail chicks' spatial exploration of a novel environment. Developmental Psychobiology, 52, 693.

Beckett, C., Maughan, B., Rutter, M., Castle, J., Colvert, E., et al. (2006). Do the effects of early severe deprivation on cognition persist into early adolescence? Findings from the English and Romanian adoptees study. Child Development, 77, 696-711.

Bolhuis, J.E., Schouten, W.G.P., de Leeuw, J.A., Schrama, J.W. \& Wiegant, V.M. (2004). Individual coping characteristics, rearing conditions and behavioural flexibility in pigs. Behavioral Brain Research, 152, 351-360.

Brillaud, E., Morillion, D., \& de Seze, R. (2005). Modest environmental enrichment: Effect on radial maze validation and well being of rats. Brain Research, 1054, 174-182.

Burchinal, M., \& Appelbaum, M. I., (1991). Estimating individual developmental functions: Methods and their assumptions. Child Development, 62, 23-43.

Cooper, R. M., \& Zubek, J. P. (1958). Effects of enriched and restricted early environments on the learning ability of bright and dull rats. Canadian Journal of Psychology, 12, 159-164.

Debonne, M., Baarendse, P. J. J., van den Brand, H., Kemp, B., Bruggeman, V., \& Decuypere, E. (2008). Involvement of the hypothalamic-pituitary-thyroid axis and its interaction with the hypothalamic-pituitary-adrenal axis in the ontogeny of avian thermoregulation: A review. World's Poultry Science Journal, 64, 309-321.

Francois, N., Mills, A. D. \& Faure, J. M. (1998). Place preferences of Japanese quail given a permanent choice between a social or a non-social but enriched situation. Behavioural Processes, 43, 163-170.

Freire, R., Cheng, H.-W. \& Nicol, C. J. (2004). Development of spatial memory in occlusion-experienced domestic chicks. Animal Behaviour, 67, 141-150.

Gottlieb, G. (2007). Probabilistic epigenesis. Developmental Science, 10(1), 1-11.

Hall F. S., Humby T., Wilkinson L. S., Robbins T. W. (1997a) The effects of isolationrearing of rats on behavioural responses to food and environmental novelty. Physiology \& Behavior, 62, 281-90. 
Hall F. S., Humby T., Wilkinson L. S., Robbins T. W., (1997b) The effects of isolationrearing of rats on preference by rats for a novel environment. Physiology \& Behavior, 62, 299-303.

Hawkins P., Morton, D. B., Cameron, D., Cuthill, I., Francis, R., et al. (2001). Laboratory birds: Refinements in husbandry and procedures. Fifth report of the BVAAWF/FRAME/RSPCA/UFAW Joint Working Group on Refinement. Laboratory Animals, 35.

Hock, R. R. (2009). Forty studies that changed psychology: Explorations into the history of psychological research. Upper Saddle Rive, NJ: Pearson Prentice Hall.

Hazard D., Couty M., Faure J.M., \& Guémené D. (2005). Daily and photoperiod variations of hypothalamic-pituitary-adrenal axis responsiveness in Japanese quail selected for short or long tonic immobility. Poultry Science Association, 84, 19201925.

Janus, C., Koperwas, J. S., Janus, M., \& Roder, J. (1995). Rearing environment and radial maze exploration in mice. Behavioural Processes, 34, 129-140.

Jones, B. R. (2002). Role of comparative psychology in the development of effective environmental enrichment: Strategies to improve poultry welfare. International Journal of Comparative Psychology, 15, 77-106.

Lazic, M., Schneider, S. M., \& Lickliter, R. (2007). Enriched rearing facilitates spatial exploration in northern bobwhite (Colinus virginianus) neonates. Developmental Psychobiology, 49, 548-551.

Lickliter, R., Dyer, A. B., McBride, T., (1993) Perceptual consequences of early social experience in precocial birds. Behavioural Processes, 30, 185-200.

Miller, K. A. \& Mench, J. A. (2005). The differential use and effects of four types of environmental enrichment on the activity budgets, fearfulness, and social proximity preference of Japanese quail. Applied Animal Behaviour Science, 95, 169-187.

Molina-Hernandez, M., Tellez-Canatara P., \& Perez-Garcia J. (2001). Isolation rearing Induced fear-like behavior without affecting learning abilities of Wistar rats. Progress in Neuro-Psychopharmacology and Biological Psychiatry 25, 11111123

Rosenzweig, M. R., Bennett, E. L., \& Diamond, M. C. (1972). Brain changes in response to experience. Scientific American, 226, 22-29.

Sackett, G. P. (1965). Effects of rearing conditions upon the behavior of rhesus monkeys. Child Development, 36, 855-868. 
Sanchez, M.M., Hearn, E.F., Do, D., Rilling, J.K., \& Herndon, J.G. (1998). Differential rearing affects corpus callosum size and cognitive function of rhesus monkeys. Brain Research, 812, 38-49.

Schrijver, N. C., Bahr, N. I., Weiss, I. C., \& Wurbel, H. (2002). Dissociable effects of isolation rearing and environmental enrichment on exploration, spatial learningand HPA activity in adult rats. Pharmacology, Biochemistry, and Behavior, 73, 209-224.

Valance, D., Boissy, A., Després, G., Arnould, C., Galand, C., Favreau, A., et al. (2008). Changes in social environment induce higher emotional disturbances than changes in physical environment in quail. Applied Animal Behaviour Science, $112,307-320$.

Vandenheede M. \& M.F. Bouissou M.F. (1998). Effects of an enriched environment on subsequent fear reactions of lambs and ewes. Developmental Psychobiology, 33, $33-45$.

van Hoek, C. S., \& King, C. E. (1997). Causation and influence of environmental enrichment on feather pecking of the crimson-bellied conure (Pyrrhura perlata perlata). Zoo Biology 16, 161-72

van Praag, H., Kempermann, G., \& Gage, F.H., (2000). Neural consequences of environmental enrichment. Nature Reviews: Neuroscience. 1, 191-198.

Whittow, G. C., \& Tazawa, H. (1991). The early development of thermoregulation in birds. Physiological Zoology, 64(6), 1371-1390.

Zhu S., Yee B. K., Nyffeler M., Winblad B., Feldon J., \& Mohammed A. H. (2006). Influence of differential housing on emotional behaviour and neurotrophin levels in mice. Behavioral Brain Research 169, 10-20. 